\title{
Aquatic Environments and Spectral Sensitivity of Rod Visual Pigments in the Retina of Rainbow Trout (Oncorhynchus mykiss): A Microspectrophotometric Study
}

\author{
Embark, H. M. ${ }^{1}$ and Hawryshyn, C. W. ${ }^{2}$ \\ ${ }^{1}$ Department of Animal Physiology, Faculty of Vet. Medicine, South Valley \\ University, Qena, Egypt \\ ${ }^{2}$ Department of Biology, Queen's University, Kingston, Ontario, Canada
}

\begin{abstract}
The vertebrate retina is a part of the central nervous system (CNS) that can be visualized in a non-invasive manner. The retina is a thin multi-layered sensory tissue of neural cells that contains millions of photoreceptors. There are two physiologically distinct types of photoreceptors across the retina, the cones and the rods. The light sensitivity of the photoreceptors results from the presence of visual pigment molecules in the outer segment of the cone and rod cells. All visual pigments are composed of two components: retinal (vitamin A aldehyde), termed a chromophore, bound to a protein called opsin. The spectral absorption properties of a visual pigment are mainly determined by the amino acid sequence of the opsin protein and the type of chromophore $\left(\mathrm{A}_{1}\right.$ or $\mathrm{A}_{2}$ or mixed) bound to the opsin. There is a shifting from $\mathrm{A}_{1}$ - to $\mathrm{A}_{2}$-based chromophores in the same opsin due to different environmental factors, e.g. aquatic environments. $\mathrm{A}_{1} / \mathrm{A}_{2}$ visual pigment ratios play a dominant role in determining rod $\lambda_{\max }$ in rainbow trout (Oncorhynchus mykiss), shifting the $\lambda_{\max }$ of the visual pigment and spectral bandwidth of absorbance (HBW, half-band width). The spectral absorption characteristics of the retinal rod photoreceptors in the Rainbow Trout (Oncorhynchus mykiss) were measured using the charge-coupled device (CCD)- based microspectrophotometer (MSP) system. We investigated retina preparations of juvenile rainbow trout (Oncorhynchus mykiss) using 54 rod photoreceptors. The spectral sensitivity of each rod photoreceptor was calculated using $\lambda_{\max }$ (wavelength of maximum absorbance) and HBW. The mean wavelength of maximum absorbance values for the alpha-bands were $503.5 \pm 2.5 \mathrm{~nm}$ for rhodopsin- dominated rods, $523.1 \pm 7.9 \mathrm{~nm}$ for porphyropsin-dominated rods, and $509.6 \pm 1.9 \mathrm{~nm}$ for rhodopsin porphyropsin mixed rods. The half-band width (HBW) of the main absorption bands was $4567 \pm 771 \mathrm{~cm}-1$ for vitamin $\mathrm{A}_{1}$-based visual pigments and $4697 \pm 487 \mathrm{~cm}-1$ for vitamin $\mathrm{A}_{2}$-based visual pigments.
\end{abstract}


Our results provide the evidence of shifting the $\lambda_{\max }$ of the visual pigment to a longer wavelength, broadening spectral bandwidth of absorbance, and presence three fundamentally different classes of rod photoreceptors in the retina of the rainbow trout depending on $\mathrm{A}_{1} / \mathrm{A}_{2}$ visual pigment ratios due to different environmental variables, e.g. aquatic environments.

KEYWORDS: Rod Photoreceptor, Spectral absorption, CCD-based microspectrophotometer, Rainbow Trou

\section{Introduction}

The Salmoniformes, also known as salmonids, are among the more primitive groups of teleost fish (one of ray-finned fish) (Kato, 1991). Salmonids, including salmon and trout, comprise a large group of closely related species with a variety of habitats, feeding strategies, and life history strategies (Ramsden et al, 2003). The variety of habitats individuals experience, often including lake, stream, and marine environments, create different challenges for the growing salmonid visual system. Changes in visual function associated with changing habitat have been reported in several species of teleost fishes (Shand et al, 2002). The rainbow trout (Oncorhynchus mykiss) is a species of salmonid native to cold-water tributaries of the Pacific Ocean in Asia and North America (Scott and Crossman, 1973). It migrates to fresh water to spawn, and either return to the ocean or further down stream after reproducing.

The teleost retina has various properties, including a layered structure, a regular mosaic of photoreceptors, and continuous growth throughout life (Lyall, 1957). The retina is a thin multi-layered sensory tissue of neural cells that lines the back of the inside of the eye globe. The retina contains millions of photoreceptors (rods and cones) that capture light rays, convert them into electrical impulses, and transmit images along the optic nerve to the brain. The retina contains approximately 6 million cones and 125 million rods. The cones cells are contained in the center of the retina (called the macula), the portion of the retina responsible for focused (central) and color vision. They are present throughout the retina but are most densely packed within the fovea, which is the very center portion of the macula. Cones are used at bright light and allow us to appreciate color. The rod cells are spread throughout the peripheral retina and function best in dim lighting. They are responsible for peripheral and night vision.

The light sensitivity of the photoreceptors results from the presence of visual pigment molecules contained within a specialized region of the cell, called the outer segment. All vertebrate 
visual pigments are composed of two components: retinal (vitamin A aldehyde), termed a chromophore, bound to a protein called opsin (Temple et al, 2006). Different visual pigments have different opsins, and this accounts for the variations in their color sensitivity. The light sensitivity of the visual pigments is due to the retinal chromophore. One rod pigment called rhodopsin, and three cone pigments which absorb light maximally in the red, green and blue regions of the spectrum, are in the human retina; non-mammalian species may have 4 or more cone visual pigments (MacNichol, 1986). The spectral absorption properties of a visual pigment are mainly determined by the amino acid sequence of the opsin protein and the type of chromophore bound to the opsin. Variations in amino acid sequence account for different classes of vertebrate opsins and thus the wavelength of maximum absorption $\left(\lambda_{\max }\right)$ of the visual pigment. However, some vertebrates, referred to as "paired pigment" or "labile pigment pair" species (Dartnall and Lythgoe, 1965; Allen and Munz, 1983), are capable of shifting between visual pigments based on vitamin $A_{1}$ (retinal) and vitamin $\mathrm{A}_{2}$ (3,4-dehdyroretinal). Shifting from $\mathrm{A}_{1^{-}}$to $\mathrm{A}_{2}$-based chromophores in the same opsin has the effect of shifting the $\lambda_{\max }$ of the visual pigment to a longer wavelength, broadening spectral bandwidth of absorbance (HBW, half-band width), and decreasing the molar extinction coefficient (Wald and Brown, 1953; Brown et al, 1963; Bridges, 1967).

Microspectrophotometry (MSP) is a well-established and effective method for studying the spectral absorption properties of individual photoreceptors. In the early 1960s, the microspectrophotometry has emerged as a valuable technique for the optical recordings from vertebrate photoreceptors (Marks and MacNichol, 1962; Marks, 1965; Liebman and Entine, 1968). Since then Scientists have been successful in utilizing MSP to characterize the spectral absorption properties of photoreceptors over a broad range of animals (e.g. Bowmaker, 1984; Bowmaker, 1990; Loew, 1995; Sillman et al., 1997). MSP in comparative biology has played a major role in advancing our knowledge about taxonomic and functional of individual photoreceptors in vertebrates and understanding the ultraviolet (UV) photoreception (Avery et al., 1983; Hart et al, 1998).

Most MSP systems used in examining vertebrate photoreceptors are designed to measure the transmitted light flux through photoreceptor outer segment (sample spectrum) and a clear area of the field (reference spectrum), as the spectrum is scanned (at $300-800 \mathrm{~nm}$ wavelength). With the rapid development of electro-optical technology, CCD (charge-coupled device) chip technology has been used recently with success in a wide 
variety of spectroscopic applications (Tang et al, 1994). One of the important features of CCD chip technology is that it is comparatively fast $(800 \mathrm{~ms}$ per scan and only one scan required) relative to wavelength-scanning MSP systems (up to 10 s per scan with multiple scans but there are appreciable differences between each MSP apparatus). This is possible because CCD-MSP system uses one flash of full-spectrum light (300-800nm) with a short-duration $(800 \mathrm{~ms})$. The transmitted flux is measured by a CCD detector used in tandem with a high-resolution $(0.4 \mathrm{~nm})$ spectrograph, providing the same information as a MSP system in a much shorter period of time (Hawryshyn et al., 2001).

In this paper, we describe and examine the rod visual pigment absorbance in the retina of the rainbow trout (Oncorhynchus mykiss) using CCD-based MSP instrument. The goal of this study was to investigate the spectral sensitivity of retinal rod photoreceptors in rainbow trout (Oncorhynchus mykiss) and tracked the change in $\lambda_{\max }$ as a correlate of $\mathrm{A}_{1} / \mathrm{A}_{2}$ ratio in rod photoreceptors.

\section{Materials and methods}

\section{Experimental Animals and care}

Fish, juvenile rainbow trout Oncorhynchus mykiss (Walbaum), were collected from the Rainbow Springs Trout Hatchery (Thamesford, Ontario, Canada). They were held at a mean water temperature of $15 \pm 1^{\circ} \mathrm{C}$ in constantly flowing aerated water and a photoperiod of $12 \mathrm{~h}: 12 \mathrm{~h}$ light:dark cycle fluorescent lighting in the Aquatic Facility of the Queen's University. Fish were fed trout pellets on a daily basis, ad libitum, of a commercial trout food (Classic Sinking Feed, Martin Mills, Elmira, Ontario, Canada). All fish care and procedures were performed in accordance with the Queen's University Animal Care Committee, under the auspices of the Canadian Council for Animal Care regulations.

\section{Tissue preparation}

Fish were dark-adapted overnight, then sacrificed by an overdose of $300 \mathrm{mgl}^{-1}$ MS-222 (Tricaine Methanosulphonate, Cresent Research Chemicals, Pheonix, Arizona, USA), followed by cervical transection. The eyes were enucleated and hemisected along an anterior posterior axis under near infrared illumination provided by light-emitting diodes (LEDs) with a peak output at $660 \mathrm{~nm}$. The retinae were removed using forceps and placed in a cell culture dish containing freshly prepared, icechilled Minimum Essential Medium (MEM; Sigma). 292 $\mathrm{mgl}^{-1} \quad$ Lglutamine and $350 \mathrm{mgl}^{-1} \mathrm{NaHCO}_{3}$ were added, the $\mathrm{pH}$ adjusted to 7.5 using $1 \mathrm{moll}^{-1} \mathrm{NaOH}$, and the solution filtered through a $0.22 \mu \mathrm{m}$ filter. The dish was kept in a lighttight container placed on ice. Illumination was provided by infrared LEDs. A piece of retina 1- 
$2 \mathrm{~mm}^{2}$ was sectioned using a razor blade segment under infrared LEDs and placed on a glass coverslip. A razor blade was used to cut the retina section into many small pieces. This provided better preparations than could be attained by teasing the tissue with forceps. A drop of MEM (Minimal Essential Media, Sigma, $\mathrm{pH}$ adjusted to 7.47.6) was applied to the sample. A second coverslip was placed over the sample and sealed with paraffin.

\section{Microspectrophotometry Recording}

The MSP recordings were performed as reported by Govardovskii et al (2000) with an instrument modified by Hawryshyn et al (2001). The CCD-MSP instrument used in this study has been described in detail in Hawryshyn et al (2001). Fig. 1 shows a schematic layout of the experimental apparatus. Briefly, a short duration (0.8 to $1.2 \mathrm{~s})$ beam light of full spectrum (300-800nm), unpolarized light was provided by a 150W xenon arc lamp (Oriel, Stratford, Connecticut, USA) and projected as a small beam through the region of interest (ROI) of the photoreceptor. The measurement beam is approximately $1 \times 2 \mu \mathrm{m}$ in size (Fig. 2, adjusted by an $X-Y$ variable slit system, Leitz) when it traverses the ROI of the photoreceptor. After beam light has passed through the retinal specimen, the transmitted light enters the spectrograph (300-nm-blazed grating, Acton Research Co, MA) and onto a Peltier cooler $\left(-50^{\circ} \mathrm{C}\right)$, back-illuminated, CCD-detector (Princeton Instruments, Roper Scientific, Inc., Trenton, NJ). The CCD array used a chip with a pixel array of 1340 columns and 400 rows. The spectral absorbance of a photoreceptor $\left[\begin{array}{ll}\log _{10} & \left.\left(I_{R} / I_{M}\right)\right] \\ \text { was }\end{array}\right.$ calculated by comparing transmitted intensity through the ROI referred to as the photoreceptor measurement $\left(I_{M}\right)$ to the transmitted intensity through an area free of debris and in media adjacent to the ROI referred to as the reference measurement $\left(I_{R}\right)$.

The retinal specimen was examined under infrared illumination using Ealing Far Red, Schott RG850 to prevent bleaching of photoreceptors. An infrared camera (Canadian Photonics Laboratory), mounted on the trinocular of the inverted microscope (Zeiss Axiovert TV100), was used for viewing the microscope field. This camera was used to capture infrared images of the preparation. The search image and infrared filtered beam were displayed on the computer monitor. The position of the ROI relative to the measurement beam was controlled by a motorized stage (Marhauser-Wetzlar GmbH \& Co., Germany). The path of the motorized stage was plotted onscreen to prevent repeated measurements of ROIs.

\section{Data and Statistical analysis}

For data analysis, we used a template created by Munz and Beatty (1965) for calculating percent $\mathrm{A}_{2}$ in retinal extracts. Data are provided as arithmetic means \pm 
standard deviation of the mean (SDM), $\mathrm{n}$ represents the number of photoreceptors examined. All data were tested for significance using the Student's $t$-test, and only results with $\mathrm{P}<0.05$ were considered as statistically significant.

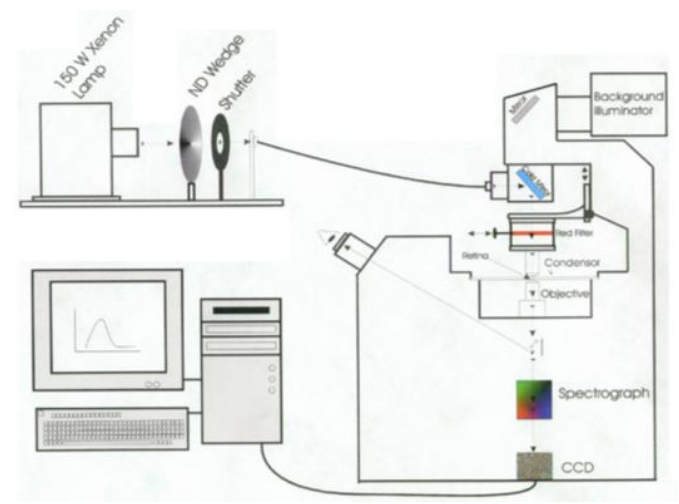

Figure 1: General diagram of the CCD microspectrophotometer. With back illumination; infrared filter; beam splitter; CCD, charge-coupled device camera; computer software; reflective objective as condenser; fibre optic cable; microscope; objective lens; xenon lamp (light source); shutter; infrared filter mounted on a swing arm; spectrograph; $X-Y$ variable aperture; $X-Y$ stage.

Figure 2: Diagram of a single beam absorption measurement for Rods. Where the measurement beam is approximately $1 \times 2 \mu \mathrm{m}$ in size. The beam is beside the target rod photoreceptors.

\section{Results}

We measured a total of 54 rainbow trout rod photoreceptors. Representative images of rainbow trout rod photoreceptors taken under Infrared illumination 
were collected when possible and presented in Fig. 3. In this study, we were able to differentiate rhodopsin, porphyropsin and mixed $A_{1}$ and $A_{2}$ visual pigment spectra.

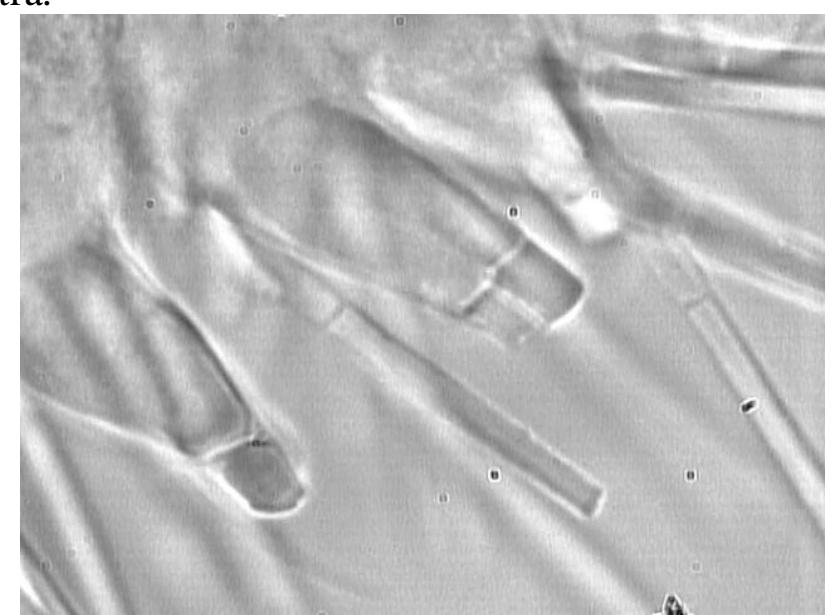

Figure 3: Representative image of rainbow trout photoreceptors. Rods and cones photoreceptors are taken under infrared illumination.

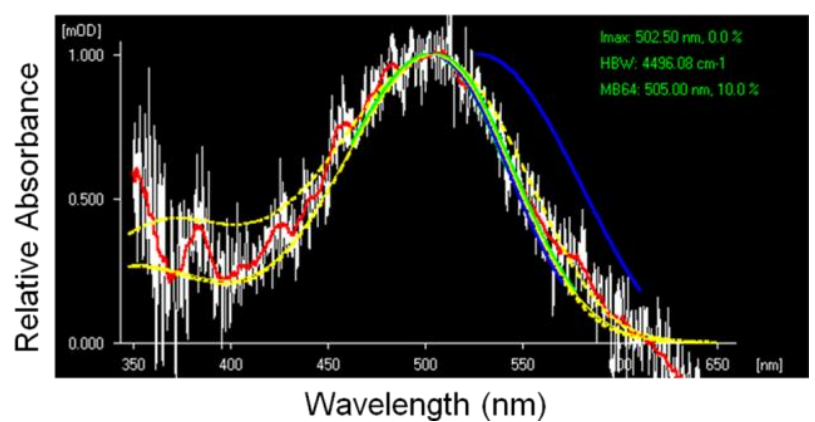

Figure 4: Normalized spectral absorbance curve from 100 percent $A_{1}$-based rods. White lines are the 1,340 raw data points collected between 300 and $800 \mathrm{~nm}$. Dark red lines are nine-point adjacent averaging smoothed lines. Green lines are the nonlinear least-squares fit of the upper 20 percent of the weighted A1/A2 averaged Govardovskii et al. (2000) template. Blue lines are the 503 and $527 \mathrm{~nm}$ Munz and Beatty (1965) template curves which are fit from $\lambda_{\text {max }}$ to a point at 20 percent of the maximum on the long wavelength arm. Thick yellow (dashed) line is the 100 percent $A_{1}$ template. Thin yellow (solid) line is the best-fit template based on the calculated $\lambda_{\max }$. Values in the upper right-hand corner are estimates based on template fitting, $\lambda_{\max }=$ Govardovskii et al. (2000) fit, and MB64 = Munz and Beatty (1965) fit, HBW $=$ half-band width of the curve at 50 percent of the peak height measured on a scale of $\mathrm{cm}^{-1}$. 


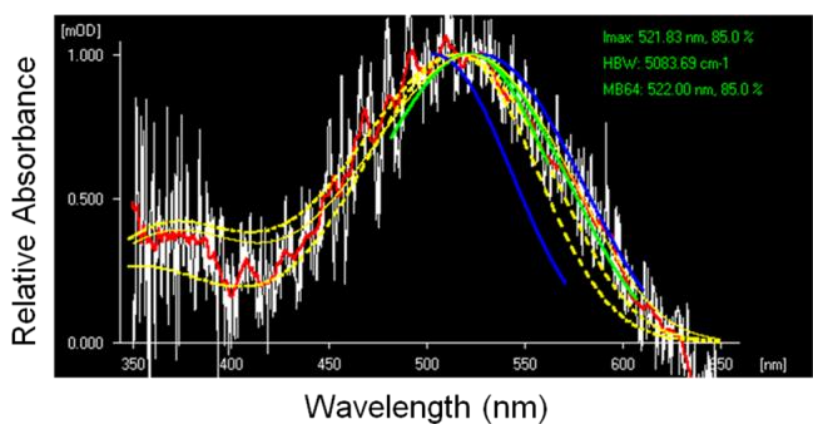

Figure 5: Normalized spectral absorbance curve from 100 percent $A_{2}$-based rods. White lines are the 1,340 raw data points collected between 300 and $800 \mathrm{~nm}$. Dark red lines are nine-point adjacent averaging smoothed lines. Green lines are the nonlinear least-squares fit of the upper 20 percent of the weighted Al/A2 averaged Govardovskii et al. (2000) template. Blue lines are the 503 and $527 \mathrm{~nm} \mathrm{Munz}$ and Beatty (1965) template curves which are fit from $\lambda_{\max }$ to a point at 20 percent of the maximum on the long wavelength arm. Thick yellow (dashed) line is the 100 percent $A_{2}$ template. Thin yellow (solid) line is the best-fit template based on the calculated $\lambda_{\max }$. Values in the upper right-hand corner are estimates based on template fitting, $\lambda_{\max }=$ Govardovskii et al. (2000) fit, and MB64 = Munz and Beatty (1965) fit, HBW $=$ half-band width of the curve at 50 percent of the peak height measured on a scale of $\mathrm{cm}^{-1}$.

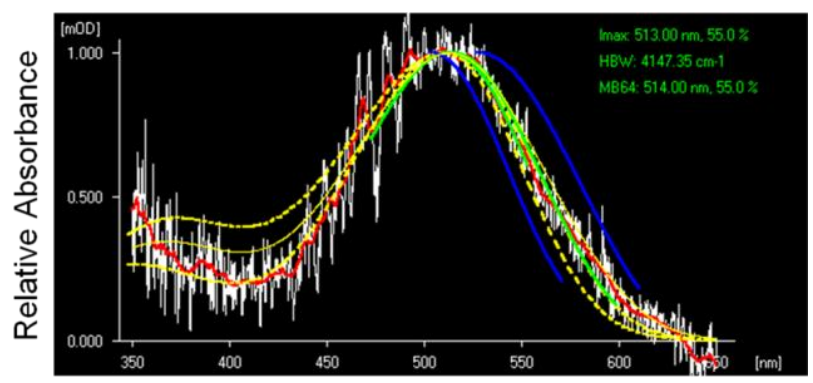

Wavelength $(\mathrm{nm})$

Figure 6: Normalized spectral absorbance curve from $A_{1} / A_{2}$ mixture rods. White lines are the 1,340 raw data points collected between 300 and $800 \mathrm{~nm}$. Dark red lines are nine-point adjacent averaging smoothed lines. Green lines are the nonlinear least-squares fit of the upper 20 percent of the weighted Al/A2 averaged Govardovskii et al. (2000) template. Blue lines are the 503 and $527 \mathrm{~nm}$ Munz and Beatty (1965) template curves which are fit from $\lambda_{\max }$ to a point at 20 percent of the maximum on the long wavelength arm. Thick yellow (dashed) line is the 50\% $A_{1}: 50 \% A_{2}$ template. Thin yellow (solid) line is the best-fit template based on the calculated $\lambda_{\max }$. Values in the upper right-hand corner are estimates based on template fitting, $\lambda_{\max }=$ 
Govardovskii et al. (2000) fit, and MB64 = Munz and Beatty (1965) fit, HBW $=$ half-band width of the curve at 50 percent of the peak height measured on a scale of $\mathrm{cm}^{-1}$.

\section{Discussion}

Our primary objective in the present study was to measure the absorbance spectra of rainbow trout photoreceptors using CCD-based MSP system and compare to those of other studies on the Pacific salmonid fishes (e.g. Hawryshyn et al., 2001). Data derived from the CCD-based MSP system compare favourably in $\lambda_{\max }$ and HBW. In addition, CCD-based MSP was used to investigate the spectral sensitivity of retinal rod visual pigments and tracked the change in $\lambda_{\max }$ as a correlate of $\mathrm{A}_{1} / \mathrm{A}_{2}$ ratio in rainbow trout (Oncorhynchus mykiss) rod photoreceptors.

The relationship between $\lambda_{\max }$, HBW of the $\alpha$-absorption band and the $\mathrm{A}_{1} / \mathrm{A}_{2}$ ratio is not well understood for paired-pigment fishes (Hawryshyn and Hárosi, 1994). We were successful in resolving $A_{1}$ and $A_{2}$ visual pigment spectra for the rods. Govardovskii et al. (Govardovskii et al., 2000) has recently established universal templates for $\mathrm{A}_{1^{-}}$and $\mathrm{A}_{2}$-based visual pigments; however, other works (most notably by Hárosi, 1994; MacNichol, 1986) have shown that universal templates do not necessarily account for all absorbance curves with $\lambda_{\max }$ values throughout the spectrum.

Using long-wavelength limb template-fitting, we have been able to determine the $A_{1} / A_{2}$ ratio of rods from a wide size range of rainbow trout to within $10 \%$ of the $\mathrm{A}_{1} / \mathrm{A}_{2}$ ratio. Thus, the rod absorbance spectra could be resolved into those conforming to $\mathrm{A}_{1}, \mathrm{~A}_{2}$ and mixed $\mathrm{A}_{1} / \mathrm{A}_{2}$ visual pigment spectral properties. The absorbance spectra for the rod visual pigments yielded three different mean $\lambda_{\max }$ values, indicative of $\mathrm{A}_{1^{-}} \quad\left(\lambda_{\max }\right.$ 503.5 $\pm 2.5 \mathrm{~nm}), \quad \mathrm{A}_{2}$-based $\quad\left(\lambda_{\max }\right.$ $523.1 \pm 7.9 \mathrm{~nm})$ and mixed $\mathrm{A}_{1} / \mathrm{A}_{2}$ $\left(\lambda_{\max } 509.6 \pm 1.9 \mathrm{~nm}\right)$ rod visual pigments (Fig. 4-6). Further refinement of this technique of accurately estimating the $\mathrm{A}_{1} / \mathrm{A}_{2}$ ratio of rods will assist in determining the smoltification status of Pacific salmonid fishes (Alexander et al., 1994; and Alexander et al., 1998).

In accordance with previous studies, we found that the change in $\operatorname{rod} \lambda_{\max }$ in rainbow trout (Oncorhynchus mykiss) matched that predicted for a change in vitamin $A_{1} / A_{2}$ visual pigment composition. Our results provide an evidence to support the notion that $A_{1} / A_{2}$ visual pigment ratios play a dominant role in determining $\operatorname{rod} \lambda_{\max }$ in rainbow trout (Oncorhynchus mykiss) due to different environmental variables.

Many species of salmon are anadromous (migrate from fresh water into the sea) and change their visual pigment composition from a 
porphyropsin (freshwater visual pigment) to rhodopsin (marine visual pigment) before their seaward migration (Hawryshyn et al., 2001). Investigating adaptive changes in visual pigment composition of rods over time, together with variation in other physiological properties (modification of gill epithelia, silvering), are now being examined as tools for estimating the smoltification event of teleost fishes. One main aspect of the application of study results is to use retinal visual pigment composition as an index of smolt status in a purpose that may have an embodiment of importance for enhancement hatchery fish stocking Programs and the aquaculture fishing industry.

\section{Acknowledgements}

The authors acknowledge all members and professors of the department of Animal Physiology, Faculty of Veterinary Medicine, South Valley University and of the department of biology, Queen's University, Kingston, Ontario, Canada, for their constant encouragement and kind help.

\section{References}

Avery, J. A., Bowmaker, J. K., Dzamgoz, M. B. A. and Downing, J. E. (1983): Ultraviolet sensitive receptors in a freshwater fish. J. Physiol. Lond. 334, 23P

Bowmaker, J. M. (1984): Microspectrophotometry vertebrate photoreceptors. Vision Res. 24, 1641-1650

Bowmaker, J. M. (1990): Visual pigments of fish. In The Visual System of Fish (ed. R. H. Douglas and M. B. A. Djamgoz), pp. 81107. London: Chapman and Hall

Govardovskii, V. I., Fyhrquist, N., Reuter, T., Kuzmin, D. G. and Donner, K. (2000): In search of a visual pigment template. Vis. Neurosci. 17, 509-528

Hárosi, F. I. (1994): An analysis of two spectral properties of vertebrate visual pigments. Vision Res. 11, 1359-1367

Hart, N. S., Partridge, J. C. and Cuthill, I. C. (1998): Visual pigments, oil droplets and cone photoreceptor distribution in the European starling (Sturnus vulgaris). J. Exp. Biol. 201, 14331446

Hawryshyn, C. W., Haimberger, T. J. and Deutschlander, M. E. (2001): Microspectrophotometric measurements of vertebrate photoreceptors using CCD-based detection technology. J. Exp. Biol. 204, 2431-2438

Kato, F. (1991): Life histories of masu and amago salmon (Oncorhynchus masou and Oncorhynchus rhodurus). In: Groot, C.; Margolis, L., eds. Pacific salmon life histories. Vancouver, BC: University of British Columbia Press: 447-520

Liebman, P. A. and Entine, G. (1968): Visual pigments of frog and tadpole (Rana pipiens). Vision Res. $8,761-775$ 
Loew, E. R. (1995): Determinants of visual pigment spectral location and photoreceptor cell spectral sensitivity. In Neurobiology and Clinical Aspects of the Outer Retina (ed. M. B. A. Djamgoz, S. N. Archer and S. Vallerga), pp. 57-78. London: Chapman \& Hall

Lyall, A. H. (1957): The growth of the trout retina. Q. J. Microsc. Sci. 98, 101- 110

MacNichol, E. F. (1986): A unifying presentation of photopigment spectra. Vision Research 26, 1543-1556

Marks, W. B. (1965): Visual pigments in single goldfish cones. J. Physiol. Lond. 178, 14-32

Marks, W. B. and MacNichol, E. F., Jr (1962): Bleaching spectra of single goldfish cones. Fed. Proc. 519, Abstract 2143

Munz, F. W. and Beatty, D. D. (1965): A critical analysis of the visual pigments of salmon and trout. Vision Res. 5, 1-17

Ramsden, S. D., Brinkmann, H., Craig W. Hawryshyn, C. W. and John S. Taylor, J. S. (2003): Mitogenomics and the sister of Salmonidae. TRENDS in Ecology and Evolution 18, 607-610

Scott, W. B. and Crossman, E. J. (1973): Freshwater fishes of
Canada. Fisheries Research Board of Canada Bulletin. 184, 1-966

Shand, J., Hart, N. S., Thomas, N. and Partridge, J. C. (2002): Developmental changes in the cone visual pigments of black bream Acanthopagrus butcheri. J. Exp. Biol. 205, 3661-3667

Sillman, A. J., Govardovskii, V. I., Röhlich, P., Southard, J. A. and Loew, E. R. (1997): The photoreceptors and visual pigments of the garter snake (Thamnophis sirtalis): a microspectrophotometric, scanning electron microscope and immunocytochemical study. J. Comp. Physiol. A 181, 89-101

Tang, Z., Ho, R., Xu, Z., Shao, Z. and Somlyo, A. P. (1994): A highsensitivity CCD system for parallel electron energy-loss spectroscopy (CCD for EELS). J. Microsc. 175, 100-107

Temple, S. E., Plate, E. M., Ramsden, S., Haimberger, T. J., Roth, W. M. and Hawryshyn, C. W. (2006): Seasonal cycle in vitamin A1/A2-based visual pigment composition during the life history of coho salmon (Oncorhynchus kisutch). J Comp. Physiol. A 192, 301-313 